\title{
Epidemiology of Talaromycosis (Penicilliosis) Marneffei in India: An Update
}

\author{
Harish C. Gugnani, Neelam Sood
}

\section{ABSTRACT}

Talaromycosis marneffei (Penicilliosis marneffei) caused by a thermally dimorphic fungus, Talaromyces (Penicillium) marneffei is emerging as an important systemic opportunistic mycosis in HIV infected patients in several countries in Southeast Asia and in northeast India. This study presents an update on epidemiological and clinical aspects of talaromycosis marneffiei in India. A thorough search of literature was done in Medline, PubMed, and Google Scholar, for accessing relevant data. Fifty-three cases of this disease have originated from the Manipur State. Several cases have been reported from Assam, a few are also known from Meghalaya, Mizoram, Nagaland, Sikkim, Delhi and Maharashtra. Clinical manifestations of disseminated infection in AIDS patients are fever, anemia, weight loss, weakness, lymphadenopathy, hepatosplenomegaly, respiratory signs, and characteristic skin lesions. The bamboo rat (Cannomys badius) has been found to be a natural host of Talaromyces (Penicillium) marneffei in Manipur. It is noteworthy that one of the ten T. marneffei isolates from bamboo rats shared the genotype with a human isolate from that area providing evidence for common source of infection for the rats and humans and host to host transmission. Natural reservoir of $T$. marneffei has not been established, though it has been demonstrated that the fungus can survive in sterile soil for several weeks, but only for a few days in unsterile soil. There is need for comprehensive investigation of $T$. marneffei infection in humans, and for occurrence of the fungus in bamboo rats and in soils of the rat burrows in India employing conventional and molecular techniques.

Keywords: Talaromycosis marneffei, epidemiological and clinical aspects, India, update.

Published Online: September 16, 2020

ISSN: $2593-8339$

DOI: $10.24018 /$ ejmed.2020.2.5.478

Harish C. Gugnani *

Vallabhbhai Patel Chest Institute, University of Delhi, India.

(e-mail: harish.gugnani@ ${ }^{@}$ gmail.com)

\section{Neelam Sood}

Deen Dayal Upadhaya Hospital, India. (e-mail: neesu123@hotmail.com)

*Corresponding Author

\section{INTRODUCTION}

Talaromycosis marneffei (Penicilliosis marneffei) is caused by a thermally dimorphic fungus, Talaromyces (Penicillium) marneffei, a member of the family Trichocomaceae, order Eurotiales, class Eurotiomycetes of division Ascomycota. It is the only dimorphic species in the genus Talaromyces [25]. Infection caused by Talaromycosis marneffei is emerging as an important systemic opportunistic infection, being potentially life-threatening in $\mathrm{HIV}+$ patients with low CD4 counts [32]. Talaromycosis marneffei is geographically restricted to Southeast Asian countries such as Thailand, Vietnam, Hong Kong, Taiwan, Philippines, Malaysia, Myanmar, Cambodia, Laos, and Northeast India. In northern Thailand, it is the third commonest opportunistic infection after tuberculosis and cryptococcosis in HIV-infected patients [32]. A large number of cases have also occurred in non-HIV-infected patients [6]. This infection is rare in Europe and all the cases described were residents or had stayed in an endemic area.

Talaromyces marneffei was first isolated in 1956 from the hepatic lesions of a bamboo rat (Rhizomys sinensis) in Vietnam, the rat had died spontaneously from the infection
[4]. The first case of human infection was reported in 1973 from a patient (an American priest) with Hodgkin lymphoma who lived in Southeast Asia [7]. T. marneffei grows in the infected tissues and at $37{ }^{\circ} \mathrm{C}$ (body temperature) as a fission yeast i.e. arthroconidia formed from hyphae. At room temperature $\left(25-30{ }^{\circ} \mathrm{C}\right)$ it grows as a filamentous form (mold) and produces a diffusible red pigment; microscopically biverticilate and monoverticillate penicillii bearing curved chains of conidia are observed. An infected patient does not act as a source of infection. The first four cases in India were of disseminated infection in $\mathrm{HIV}+$ patients from Manipur State in northeast (NE) India [29], the patients had fever, weight loss, anorexia, hepatosplenomegaly, and molluscum contagiosum like lesions on face and upper trunk.

\section{MATERIALS AND METHODS}

A thorough search of lite6ature was done in Medline, PubMed, Biomed lib, Cochrane library and Google Scholar using different sets of keywords, viz. talaromycosis marneffei, Talaromyces marneffei, rodent carriers, environmental reservoir, for accessing relevant data from 
different states of India. Cross references in the collected research publications were also accessed extracting relevant information.

\section{RESULTS AND DISCUSSION}

\section{A. Geographic distribution of cases}

Talaromycosis marneffei is geographically limited to Southeast Asian countries, viz. Hong Kong, Thailand, Vietnam, Taiwan, China, Laos, Cambodia, Philippines', Malaysia, Myanmar and northeast India. Majority of the cases (53) in India have originated from Manipur state in NE India [8], [26], [28], [33]. Twelve autochthonous cases have been reported from Assam [3]. [9], [19]. [21], [24], [25], [28], [33], a state bordering Manipur and a few cases in other NE states, viz. Nagaland -1 [18] Mizoram-2 [18], [30], and Meghalaya-1 [8], Sikkim-1 [28]. Cases reported from other parts of India include two each from Kerala [27] [31] and Maharashtra [2] [34], and one from Delhi [35], In one additional case, there was history of travel to northeast India without mention of the specific state [28]. A study from Sikkim [1] reported $14.28 \%$ of 32 cases of onychomycosis due to T. marneffei but no details of the identification of the fungus in culture or tissue were mentioned, nor were these available on inquiry from the corresponding author of the article; hence identification of the etiologic agent as T. marneffei in these cases is doubtful.

B. Bamboo rats found to be natural hosts of Talaromyces (Penicillium) marneffei

Bamboo rats known to be carriers of T. marneffei in southeast Asia include Cannomys badius (The Lesser Bamboo rat), Rhizomys pruinosus (Hoary bamboo rat), $R$. sinensis (Chinese bamboo rat) $R$. sumatrensis (Large bamboo rat, Sumatran or Indo-Malayan rat [2]. In India only two species of bamboo rats, viz. $C$. badius and $R$ pruinosus are found in north eastern states, excepting Arunachal Pradesh and Nagaland [11]. Bamboo plantations harbor several species of rats other than bamboo rats. A study by Gugnani et al [10] comprehensively investigated 182 sympatric rodents comprising five species (Bandicota bengalensis, Rattus norvegicus, Rattus, Rattus niditus, Mus musculus, and indigenous reddish-brown bamboo rat Cannomys. badius from bamboo plantations in Manipur State, NE India. Ten of 110 C. badius rats were found to be asymptomatically infected with $T$. marneffei. It is noteworthy that one of the 10 isolates from bamboo rats shared the genotype with that of a human isolate from that area [10]. This provides evidence for a common source of infection for the rats and human, and host to host transmission. A similar finding has been reported by Cao et al [5] in China. The banboo rat, Cannomys badius is a very common in Manipur but is also known to occur in Arunachal Pradesh, Assam, Nagaland, Mizoram, and possibly it occurs in other States. There is no information on carriage of Talaromyces marneffei by this rat and other rodents in these States, though several indigenous human cases of T. marneffei have been recorded from Assam, and one from Nagaland.

\section{Occurrence in soil and environmental reservoir of} infection

Natural reservoir of T. marneffei has not been definitely established. In a study by Gugnani et al [12], none of the 176 soil samples collected from bamboo plantations in several parts of India, including 25 collected from the burrows of the bamboo rat (Cannomys badius) in Manipur yielded T. marneffei. However, it has been demonstrated that the fungus can survive in sterile soil for several weeks, but only for a few days in unsterile soil [15]. Cao et al [5] were able to isolate T. marneffei from 5 of 43 samples of soil collected from the burrows and surrounding environment of bamboo rats in China. Thus, the most probable environmental reservoir for infection appears to be the soil of bamboo rat burrows. There is no evidence regarding transmission of infection through direct exposure to bamboo rats, or eating bamboo rats. Since bamboo rats come in contact with bamboo plants, it would be worthwhile to investigate possible carriage of T. marneffei on parts of bamboo plants.

\section{Clinical spectrum of infections}

Commonest symptoms in HIV+ patients in order of frequency are fever, weight loss, weakness, anemia, skin lesions, and hepatosplenomegaly and lymphadenopathy as reviewed by Ranjana et al [22] and Sethuraman et al [27], there were no respiratory symptoms in these cases. Skin lesions are typically centrally umblicated generalized papules or nodules, which may be confused with molluscum contagiosum. A high index of suspicion in the presence of typical skin lesions can lead to early diagnosis. Cases of rare presentations include one of acute abdomen [22], another one with acute abdomen and mesenteric/retroperitoneal lymphadenitis [28] and the third one of total nail dystrophy [31]. Three HIV positive adult patients including two originating from Manipur [14] [18], and one from Assam [23], and another HIV positive 12year-old boy from Assam [24] with T. marneffei infection, manifested as immune reconstitution inflammatory syndrome (IRIS).

There have been 110 cases of talaomycosis marneffei in non-HIV subjects reported in world literature [6]; the major clinical features in these cases were fever, cough. malaise, anemia, thrombocytosis, cutaneous or subcutaneous lesions, lymphadenopathy, weight loss, and dyspnea. In a retrospective cohort study involving 116 HIV patients and 34 non-HIV patients infected with T. marneffei in Thailand, it was found that the non-HIV-infected patients were significantly older, less likely to have fever, splenomegaly and umbilicated skin lesions, and more likely to have Sweet's syndrome and osteoarticular lesions. [6]. The nonHIV-infected patients also had higher leukocyte, CD4 lymphocyte and platelet counts, and lower alanine transaminase level and blood culture-positive rate [6]. There are only four cases of talaromycosis marneffei in non-HIV patients from India, one a diabetic with probable early nephropathy with symptoms of chronic pneumonia, another one a post-renal transplant patient on immunosuppressive treatment both being from Sikkim [8]. The third case from Kerala [27] had a verrucose lesion on the foot with no history of trauma, tuberculosis, diabetes mellitus, HIV, hepatitis B or HCV infection, and the fourth one was an 
immunocompetent patient with keratitis from Maharashtra [33].

The incidence of HIV-associated T. marneffei infection has decreased considerably with the use of potent antiretroviral therapy (ART). However, T. marneffei infection continues to cause considerable morbidity/mortality in AIDS patients who do not seek or have access to ART, or who have a suboptimal response to HIV therapy [35]. In India, indigenous cases of Talaromyces marneffei infection are known from NE states of Manipur, Assam, Mizoram, Nagaland, and Sikkim, bur no case is known so far from Tripura. The maximum number of cases have been described from Manipur. The suggested association of the disease with rainy season [32] should be further explored. Two species of bamboo rats are found in the Northeast India, Cannomys badius, the Lesser Bamboo Rat and Rhizomys pruinosus, the larger Hoary Bamboo Rat. Cannomys badius in known to be carrier of the Talaomyces marneffei in Manipur. Carriage of $T$. marneffei by $R$. pruinosus in northeast India needs to be investigated. Further as described earlier, a few autochthonous cases of talaromycosis marneffei have been reported in the past decade from some states of India far away from the northeast, viz Delhi, Maharashtra, Tamil Nadu and Kerala. The stated species of bamboo rats are not found in these areas. Thus, an attempt should be made to investigate if other species of rats associated with bamboo plants in these areas harbor $T$. marneffei.

It is worth mentioning here that three locally acquired cases of talalromycosis marneffei in HIV infected patients have been reported from West Africa, one originating from Burkina Fasso [13], another one from Ghana [17], and third one from Togo [20]; these patients had never travelled to Southeast Asia. Cases among Africans who have travelled to endemic countries in Southeast Asia are much more common [32]. It is possible that pockets of endemic foci of T. marneffei infection exist in other countries in Africa and elsewhere.

Several other Talaromyces species including $T$. amestolkiae, T. decumbens, T. piceus and T. purpurogenus and are common in soil, rotten fruits and air and have been reported to cause superficial and invasive fungal infection in humans in USA, Mexico, Argentina, UK and Turkey [16]. It would be worthwhile investigating occurrence of human infections caused by these species in India.

Our knowledge of the natural reservoir of $T$. marneffei and mode of infection has not been definitely established. It is possible that conidiospores of the fungus from soil or from direct contact with carrier bamboo rats are inhaled and then enguled in alveolar macrophages via respiratory route.

\section{CONCLUSION}

The need for comprehensive investigation of occurrence of human infections due to T. marneffei and occurrence of the fungus in environmental sources in different parts of India cannot be overemphasized. Diagnosis of infection can be challenging since other diseases like tuberculosis and histoplasmosis share many clinical features [28]. A definitive diagnosis should be made by correct identification of the causative agent in tissue and culture as described in detail [28]. Molecular methods for diagnosing the infection at an early stage should also be explored. It has been demonstrated that MALDI-TOF mass spectrometry is a good choice for rapid, less expensive primary identification of Talaromyces species [16].

\section{REFERENCES}

[1] Adhikari L, Das Gupta A, Pal R, Singh TS. Clinico-etiologic correlates o onychomycosis in Sikkim. Indian J Pathol Microbiol 2009; 52:94-7.

[2] Baradkar V, Kumar S, Kulkarni SD. Penicillium marneffei: the pathogen at our doorstep. Indian J Dermatol Venereol Leprol 2009; 75:619-20.

[3] Bordoloi P, Nath R, Borgohain M, Huda MM, Barua S, Dutta D, et al. Subcutaneous mycoses: an aetiological study of 15 cases in a tertiary care hospital at Dibrugarh, Assam, Northeast India. Mycopathologia 2015; 179:425-35.

[4] Capponi, M, Segretain G, Sureau P. Penicillosis from Rhizomys sinensis. Bull Soci Pathol Filiales 1956; 49:418-21.

[5] Cao C, Liang L, Wang W, Luo H, Huang S, Lieu D, Xu J, Henk DA, Fisher MC. Common reservoirs for Penicillium marneffei infection in humans and rodents, China. Emerging Infect Dis 2011; 17:209-14.

[6] Kawila R, Chaiwarith R, Supparatpinyo K. Clinical and laboratory characteristics of penicilliosis marneffei among patients with and without HIV infection in Northern Thailand: a retrospective study. BMC Infect Dis 2013 Oct 5;13: 464. doi: 10.1186/1471-233413-464.

[7] DiSalvo AF, Fickling AM, Ajello L. Infection caused by Penicillium marneffei: description of first natural infection in man. Am J Clin Pathol 1973; 60:259-63.

[8] George I, Sudarsanam T, Pulimood A, Mathews M. Acute abdomen: an unusual presentation of disseminated Penicillium marneffei infection. Indian J Med Microbiol 2008; 26:180-82.

[9] Gorai S, Saha M, Madhab V, Mitra S. Talaromycosis (penicilliosis): a rare, opportunistic systemic fungal infection. Indian J Dermatol 2019; 64:331-33

[10] Gugnani H, Fisher MC, Paliwal-Johsi A, Vanittanakom N, Singh I Yadav PS. Role of Cannomys badius as a natural animal host of Penicillium marneffei in India. J Clin Microbiol 2004; 42:5070-5.

[11] www.conservationindia.org/gallery/bamboo-rat-dampa-tiger-reserveMizoram.

[12] Gugnani, HC, Paliwal-Joshi A, Rahman H, Padhye AA. et al Occurrence of pathogenic fungi in soil of burrows of rats and of other sites in bamboo plantations in India and Nepal. Mycoses 2007; 50:507-11.

[13] Guiguemde KT, Sawadogo PN, Zida A, Cisse M, Sangare I, Bamba S. First Case Report of Talaromyces marneffei Infection in HIV infected Patient in the City of Ouagadougou (Burkina Faso) Med Mycol Case Rep 2019; 26:10-12.

[14] Gupta, S., Mathur, P. Maskey, D. Singh S. Immune Restoration Syndrome with disseminated Penicillium marneffei and cytomegalovirus co-infections in an AIDS patient. AIDS Res Ther 4, 21 (2007); doi.org/10.1186/1742-6405-4-21.

[15] Joshi A, Gugnani HC, Vijayan VK. Survival of Penicillium marneffei in sterile and unsterile soil. J Mycol Med 2003; 13:211-12.

[16] Li L, Chen K, Dhungana N, Jang Y, Chaturvedi V, Desmond E. Characterization of Clinical Isolates of Talaromyces marneffei and Related Species, California, USA. Emerg Infect Dis. 2019; 25:17651768. https://dx.doi.org/10.3201/eid2509.190380.

[17] Lo Y, Tintelnot K, Lippert U, Hoppe T.Disseminated Penicillium marneffei infection in an African AIDS patient. Trans $\mathrm{R}$ Soc Trop Med Hyg 2004: 187. doi:10.1016/S0035-9203(00)90271-2.

[18] Michael JS, Abraham OC, Mathai D, Mathews MS. Varied clinical manifestations of Penicillium marneffei in patients with human immunodeficiency virus: a report from south India. Mycoses 2005; 48:120-1.

[19] Nizami AI, Rama SK, Biradar C, Akambadyar R, Chakraborty D, Handique F. Penicillium marneffei infection manifesting as immune reconstitution inflammatory syndrome (IRIS) in HIV infected soldier on HAART. J Med Dental Research 2016; 3:9-13.

[20] Patassi AK, Saka B, Landoh DE, Kotosso A. et al. First observation in a non-endemic country (Togo) of Penicillium marneffei infection in a human immunodeficiency virus-infected patient: a case report. BMC Research Notes 2013;6: http://www.biomedcentral.com/1756- 
0500/6/506.

[21] Puri P, Ramesh V, Singh A, Muralidhar S, Capoor MR. Facial eruption in a human immunodeficiency virus (HIV)- seropositive patient. Int J Dermatol 2012; 51:777-9.

[22] Ranjana KH, Priyokumar K, Singh Th J, Gupta Ch C. et al. Disseminated Penicillium marneffei infection among HIV-Infected Patients in Manipur State, India. J Infection 2002; 48:268-71.

[23] Saikia L, Nath R, Mahanta J. Penicillium marneffei infection in Assam. Indian J Dermatol Venereol 2010; 76:75-6.

[24] Saikia L, Nath R, Biswanath P, Hazarika D, Mahanta J. Penicillium marneffei infection in HIV infected patients in Nadidaaresnd immune reconstitution after treatment. Indian J Med Res 2009; 129:333-4.

[25] Samson RA, Yilmaz N, Houbraken J, Spierenburg H, Peterson S, Frisvad JC. Varga J. Phylogeny and nomenclature of the genus Talaromyces and taxa accommodated in Penicillium subgenus Biverticillium. Studies in Mycology 2011; 70:159-83.

[26] Sarkar P, Saha A, Shukla S, Two sporadic cases of Penicillium marneffei infection associated with human immunodeficiency virus infection from West Bengal: A word of caution. Sex Transm Dis 2019; 40:180-2.

[27] Sasikumari O, Jose TA, Anupama S. Case of Talaromyces marneffei skin infection in an immunocompetent adult. Acad Clin Microbiol 2018; 20:49-51.

[28] Sethuraman N, Thirunarayan MA, Gopalakrishnan R, Rudramurhty S, Ramasubramanian V, Parameshwaran A. Talaromyces marneffe outside endemic areas in India: an emerging infection with atypical clinical presentations and review of published reports from India Mycopathologia 2020: https://doi.org/10.1007/s11046-019-00420$0(0)$

[29] Singh NP, Ranjana KH, Singh YI, Singh KP, Sharma SS Kulachandra M. Indigenous disseminated Penicillium marneffei infection in the state of Manipur, India: Report of four autochthonous cases. J Clin Microbiol 1999; 37:2699- 2702.

[30] Sood N, Gugnani HC. Disseminated Penicillium marneffei infection in a Myanmar refugee from Mizoram state. Indian J Pathol Microbio 2010; 53:361-3.

[31] Sunny N, Nair SP, Justus S, Beena A. Total dystrophic onychomycosis caused by Talaromyces marneffei in a patient with acquired immunodeficiency syndrome on combined anti-retroviral therapy. Indian J Dermatol Venerol Leprol 2018; 84: 87-90.

[32] Vanittanakom N, Cooper CR, Fisher MC, Sirisanthana T. Penicillium marneffei infection and recent advances in the epidemiology and molecular biology aspects. Clin Microbiol Rev 2006; 19:95110.12345).

[33] Varghese GM, Pise G, Michael SJ, Jacob M, George R. Disseminated Penicillium marneffei infection in a human immunodeficiency virus-infected individual. J Postgrad Med 2004; 50:235-6.

[34] Vyawahare CR, Misra RN, Gandham NR, Angadi KM, Paul R. Penicillium keratitis in an Immunocompetent Patient from Pune, Maharashtra, India. J Clin Diagn Res 2014;8(7):doi:10.7860/JCDR/2014/7996.4647.

[35] www.uptodate.com/contents/diagnosis-and-treatment-of-talaromycespenicillium-marneffei-infection.

[36] Yadav S, Gupta R, Anuradha S, Makkar AM. A rare case of disseminated penicilliosis - first of its kind from North India. Indian J Pathol Microbiol 2019; 62:156-8.

[37] Yanamandra U, Anantaram J, Subramanian S, Sharma M, Hazra N, Nair V. Penicilliosis presenting as fungating skin lesion. J Infect Chemother 2011;17: 700-2.

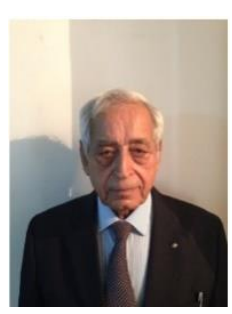

Harish C. Gugnani

Date of birth: $10^{\text {th }}$ April 1938

Place of birth: Lyallpur India.

PhD in Medical Microbiology (Delhi), FRC. Path (London).

Current position: Retired Professor of Medical Mycology, VP Chest Institute, University of Delhi, Delhi-110007, India. Current research interests include Pathogenesis, Laboratory Diagnosis and Epidemiology of Fungal infections and Infectious

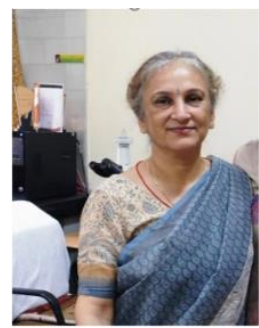

Neelam Sood

M.B.B.S. MD. (Pathology), University of Delhi, Delhi, India.

Current position: Consultant and Head of Department of Pathology \& Lab Medicine, and Director of DDU-IMS, Deen Dayal Upadhaya Hospital, Hari Nagar, New Delhi-110063, India. Current research interests include Cytology \& Histopathology, Immunochemistry in Breast Pathology, Salivary Glands, Soft Tissue, Dermatopathology.
Gynaecological pathology, and 